\title{
Managing COPD using pulmonary rehabilitation: a literature review
}

Mukundu L, Matiti MR (2015) Managing COPD using pulmonary rehabilitation: a literature review. Nursing Standard. 30, 14, 38-43. Date of submission: October 31 2014; date of acceptance: March 102015.

\begin{abstract}
This article presents a literature review of the benefits of pulmonary rehabilitation in the management of chronic obstructive pulmonary disease (COPD). Pulmonary rehabilitation has been shown to decrease patient mortality and hospital admissions. The literature search resulted in 13 articles being selected for review. Three themes were identified: improvement in exercise tolerance, improvement in breathlessness and improvement in health-related quality of life. This review enhances our knowledge of the mechanisms through which pulmonary rehabilitation benefits patients and provides an evidence base to guide nursing decisions regarding patient education, health promotion and psychological support.
\end{abstract}

\section{Authors}

Lowina Mukundu Staff nurse, Leicester Royal Infirmary, Leicester, England.

Milika Ruth Matiti Senior lecturer, College of Social Science, School of Health and Social Care, University of Lincoln, Lincoln, England. Correspondence to: Lowina04@yahoo.co.uk

\section{Keywords}

chronic obstructive pulmonary disease, COPD, literature review, pulmonary rehabilitation, qualitative research, quantitative research, respiratory care, respiratory rehabilitation

\section{Review}

All articles are subject to external double-blind peer review and checked for plagiarism using automated software.

\section{Online}

For related articles visit the archive and search using the keywords above. Guidelines on writing for publication are available at: journals.rcni.com/r/author-guidelines.

THIS LITERATURE REVIEW discusses the benefits of pulmonary rehabilitation in the management of chronic obstructive pulmonary disease (COPD). Pulmonary rehabilitation is a 'comprehensive intervention based on a thorough patient assessment followed by patient-tailored therapies that include, but are not limited to, exercise, training, education, and behaviour change, designed to improve the physical and psychological condition of people with chronic respiratory disease and to promote the long-term adherence to health-enhancing behaviours' (Spruit et al 2013). The principal goal of pulmonary rehabilitation is to achieve multidisciplinary co-ordination of a group of techniques aimed at improving the condition of patients and easing difficulties in exercise (Corhay et al 2014). Patient and family education is central to the effectiveness of pulmonary rehabilitation, involving sustained behavioural change. Patient education provides information about how pulmonary rehabilitation works, managing medication, and awareness of physiological changes in the body. It aims to enable patients to adopt behaviours which help them manage their health effectively, for example taking medications, participating in cardiovascular exercise and seeking support when necessary.

The COPD Commissioning Toolkit (NHS 2012) for commissioning pulmonary rehabilitation aims to improve the patient experience by effectively co-ordinating multidisciplinary efforts to support patients to set their own goals and take responsibility for their health, by improving access to pulmonary rehabilitation programmes. Developing effective relationships between healthcare professionals is important in patient-centred care, and to enable pulmonary rehabilitation activities to be adapted to suit the local population, according to staff availability, patient needs, availability of resources, transport arrangements and the geographical locations where pulmonary rehabilitation facilities may be established. Pulmonary rehabilitation has traditionally been led by physiotherapists; however, there is a trend for nurses to lead pulmonary rehabilitation programmes (Vincent and Sewell 2014).

COPD is a group of lung diseases characterised by progressive and irreversible airway obstruction, caused by cigarette smoke, air pollutants or genetic makeup (National Institute for Health and Care Excellence (NICE) 2015), and is predicted to be the third leading cause of death by 2030 (World Health Organization 2008). The total annual cost of COPD to the NHS is estimated to be over 
$£ 800$ million (NHS 2012). The case for pulmonary rehabilitation as an effective management strategy for COPD has been demonstrated in studies resulting in reduced hospital admissions, decreased mortality, improvements in health-related quality of life, improvements in breathlessness and improvements in exercise tolerance (Puhan et al 2011, Lan et al 2013). However, some patients with COPD have a negative perception of the effectiveness of pulmonary rehabilitation.

The aim of this article is to review the literature on the benefits of pulmonary rehabilitation and to discuss the mechanisms by which pulmonary rehabilitation activities slow the progression of COPD and foster effective self-management of COPD.

\section{Literature search}

The Scopus, Cumulative Index to Nursing and Allied Health Literature (CINAHL) and Cochrane Library databases were searched using the keywords: pulmonary rehabilitation, respiratory rehabilitation, chronic respiratory disease, COPD patients, benefits, qualitative and quantitative. Practice statements and guidelines, primary articles, systematic reviews and peer-reviewed articles published between 2008 and 2014 were included in the literature search. Studies relating to respiratory diseases other than COPD were excluded.

After screening by title, abstract, availability of articles and exclusion of duplicates, 13 articles were selected for review. A table detailing the articles selected for review is provided at: journals.rcni.com/r/copd-table. Thematic analysis identified several overlapping themes in the articles. This involved reading the articles to become familiar with the content and then rereading them with a view to generating and developing patterns and generic terms, which were grouped into themes (Boyatzis 1998). Three themes were identified: improvement in exercise tolerance, improvement in breathlessness and improvement in health-related quality of life.

\section{Improvement in exercise tolerance}

Exercise tolerance is the ability to endure physical activity involving bodily movements (Spruit et al 2013). Exercise is a physical stressor. It disrupts homeostasis and several bodily systems, primarily the respiratory system, and seeks to re-establish homeostatic balance so that the oxygen required for the generation of energy is delivered to the cells (Plowman and Smith 2014). The respiratory system and other relevant systems function effectively in good health to meet the increased energy demands associated with activity or exercise.

\section{Exercise impairment as a result of COPD}

For people with COPD, impairment of the respiratory system and other bodily systems interferes with the effective uptake and delivery of oxygen to the cells. Intolerance to exercise in these individuals is the result of an imbalance between the body's demands for energy and oxygen supply, an imbalance between ventilator capacity and requirement, weakness in peripheral muscles and defective cardiac function (Troosters et al 2010).

The flow of air breathed in by patients with COPD is limited by destruction of the alveolar walls and narrowing of the bronchioles. They may experience limited airflow at low levels of activity and at rest, in contrast to healthy individuals who experience a limited airflow at very high levels of exercise. Patients with COPD respond to the limited airflow by attempting to breathe in more air. Consequently, more air is trapped in the lungs as the breathing rate increases, because the lungs are not emptied fully on exhalation. This trapped air increases pressure in the lungs, interfering with gas exchange and affecting the cells' ability to generate the energy required for activity. Patients may feel starved of oxygen and experience fatigue, as a result of less energy being generated in the cells, and cease whatever activity they may be performing (O’Donnell and Laveneziana 2006).

Various exercise regimens and measurement tools used in pulmonary rehabilitation are aimed at slowing or reversing physical disability that may result from reduced activity or a lack of activity (Casaburi and ZuWallack 2009).

\section{Effects of pulmonary rehabilitation on exercise intolerance}

The goal of exercise in pulmonary rehabilitation is to slow progression of muscle dysfunction, thus creating chemical changes in muscle cells and increased physical ability (Man et al 2009). Man et al (2009) suggested that exercise enables the muscle to switch from fast fatiguable fibres to slow fatiguable fibres by increasing mitochondrial content and activity, which leads to efficient generation and use of substrates for glucose transportation and energy production.

A case study involving a 61-year-old woman with COPD, who was experiencing breathlessness on quickening her pace on level ground, concluded that pulmonary rehabilitation improved the patient's tolerance to exercise (Casaburi and ZuWallack 2009). In this case study, the authors suggested that, while exercise during pulmonary rehabilitation 
does not directly improve gas exchange in the lungs, it optimises the function of other bodily systems, thereby minimising lung dysfunction. This is achieved through control of cellular biochemistry, thus desensitising the brain to fatigue or breathlessness. Although this patient was not assessed in a group setting, Casaburi and ZuWallack (2009) suggested that improvement in exercise tolerance could also be explained through other, less well-defined mechanisms, such as the distraction experienced by patients when they are in groups among other patients with symptoms that are similar to or worse than their own.

Pulmonary rehabilitation provides a relevant environment for providing patient education and psychological support, leading to effective self-management of COPD. Exercise during pulmonary rehabilitation induces changes in the biochemistry of muscle cells, so that the muscles can tolerate higher levels of activity (Casaburi and ZuWallack 2009). Such chemical changes have been associated with improvement in exercise tolerance. Stav et al (2009) reported an increase in exercise endurance time in a study of 80 participants undergoing pulmonary rehabilitation, who had their exercise tolerance assessed at six-month intervals up to three years.

Casaburi and ZuWallack (2009) suggested that desensitisation may arise from social interactions, which distracts patients from the sensation of breathlessness. Participants undergoing pulmonary rehabilitation focus on achieving better exercise outcomes rather than being anxious about their breathing. Ramli and Manap (2011) reported improvement in exercise tolerance as a result of participants being less anxious and thus less breathless. Halding and Heggdal (2012) identified an improvement in exercise capacity as a result of participants being more aware of their health and using this to control breathlessness and focus on improving exercise tolerance. Therefore, patients with COPD can divert their attention and energy to their chosen task (the improvement of exercise tolerance), rather than focusing on breathlessness, with adequate education and psychological support. Access to education and psychological support contributes to patients' determination and improvement in undertaking exercise (Casaburi and ZuWallack 2009).

\section{Improvement in breathlessness}

Breathing is the mechanism whereby air flows between the atmosphere and the alveoli of the lungs (Marieb 2011). When muscles in the chest cavity relax, the volume of the chest cavity increases and the resultant low pressure causes oxygenated air to be inhaled from the atmosphere. When the muscles contract, the volume of the chest cavity is reduced and the resultant high pressure in the lungs causes air to be exhaled, along with carbon dioxide.

\section{Breathing mechanism in patients with COPD}

In patients with COPD, impairment of muscles in the chest cavity, reduced lung elasticity, defective alveoli and an inflamed airway interfere with the process of breathing and the volume of the chest cavity (Casaburi and ZuWallack 2009).

The sensation of breathlessness may be influenced by a patient's age, the effect of other illnesses and health beliefs (ZuWallack and Hedges 2008). One patient may define exertion as climbing a flight of stairs, while another may define getting out of bed and into a chair as exertion (Williams et al 2010). The subjectivity of breathlessness means it is important that nurses understand how pulmonary rehabilitation attempts to remedy breathlessness and approach pulmonary rehabilitation in a person-centred way. According to Antoniu (2010), the mechanism of breathlessness in COPD is largely the result of dynamic hyperinflation. This refers to the situation whereby the lungs do not completely empty at exhalation. This accumulation of air causes the sensation of breathing discomfort. Other factors associated with dynamic hyperinflation are neuro-chemical dysfunction, hypoxaemia, hypercapnia and a mismatch between ventilator demand and capacity (Casaburi and ZuWallack 2009).

\section{Effects of pulmonary rehabilitation on breathlessness}

Improvement in breathlessness in pulmonary rehabilitation was attributed to patient education, breathing retraining and proper use of inhalers (Lan et al 2013). Lacasse et al (2006) cited breathing retraining and patient education as important factors in improving breathlessness. Barakat et al (2008) indicated that improvement in breathlessness is achieved through breathing retraining provided by pulmonary rehabilitation; this motivates patients to tolerate the restrictive ventilator defects characteristic of COPD.

Pulmonary rehabilitation provided a group setting in which patients with COPD supported each other, challenged themselves and managed to tolerate higher levels of breathlessness (Williams et al 2010). The mechanism of psychological and social support is subjective. However, these findings confirmed the results of previous studies 
on breathlessness (Gysels and Higginson 2009). Pulmonary rehabilitation participants became more confident and coped better with breathlessness when receiving psychological support and experiencing social interaction (Halding and Heggdal 2012). Pulmonary rehabilitation offered a supportive environment in which participants learned breathing techniques, became more aware of the bodily signs associated with the onset of breathlessness and devised individual ways of self-management. Such findings emphasise the importance of pulmonary rehabilitation in providing an enabling environment in which patients learn effective self-management skills.

\section{Improvement in health-related quality of life}

Health-related quality of life, sometimes known as emotional wellbeing, refers to patients' satisfaction with life, based on their judgement of the effect of COPD on their capability to carry out activities of daily living (Pitta et al 2008). Health-related quality of life also represents patients' perception of their illness and their knowledge and understanding of the diagnosis, prognosis and management of COPD (Weldam et al 2013).

\section{COPD influence on health-related quality of life}

Factors that influence health-related quality of life in individuals with COPD are the existence of other illnesses, such as depression or high blood pressure, the severity of COPD, and the patients' age, health beliefs and coping mechanisms (ZuWallack and Hedges 2008, Riario-Sforza et al 2010).

\section{Effects of pulmonary rehabilitation on health-related quality of life}

Pulmonary rehabilitation provides an opportunity to meet the needs of patients with COPD, by providing relevant information and knowledge (de Sousa Pinto et al 2013). Patients with COPD may define health-related quality of life in accordance with the severity of their disease, as indicated by frequency of exacerbations and use of healthcare services (Cecins et al 2008, Martín et al 2008) or by knowledge of their illness (Weldam et al 2013). Gysels and Higginson (2009) showed that patients obtained information about COPD and pulmonary rehabilitation from the internet, family and friends. Halding and Heggdal (2012) suggested that nurses represent an appropriate information resource, providing knowledge and correcting misconceptions generated from information obtained from friends or the internet.
Barakat et al (2008) established that pulmonary rehabilitation reduces depression and enhances quality of life for patients with COPD. Coventry et al (2013) demonstrated that pulmonary rehabilitation reduces anxiety and depression in patients with COPD, although they were unable to identify any particular aspect of pulmonary rehabilitation as being directly responsible for the reduction in anxiety and depression. Health-related quality of life is subjective and multi-factorial. However, it would seem reasonable to credit the pulmonary rehabilitation programme for the overall improvement in health-related quality of life experienced by participants.

\section{Discussion}

The literature review presents an outcome-focused approach to pulmonary rehabilitation, rather than an exercise-based presentation. Evidence from the studies selected for this review suggest that patient education and exercise complement each other in the context of pulmonary rehabilitation, such that knowledge obtained through education motivates patients to participate in exercise, which initiates positive change. This is in keeping with the requirements of The Code. Professional Standards of Practice and Behaviour for Nurses and Midwives (Nursing and Midwifery Council (NMC) 2015) for nurses to involve patients in decisions regarding their care, by presenting information in a way that patients understand.

This review discussed the physiology of the body in health, the effects of COPD on health and the corrective mechanisms achieved through pulmonary rehabilitation. This approach enables an understanding of how pulmonary rehabilitation improves quality of life for patients with COPD, and provides important information for nurses, useful for delivering evidence-based patient education (NMC 2015).

Improvements in exercise tolerance, breathlessness and health-related quality of life are presented as separate themes. However, the benefits of pulmonary rehabilitation in COPD are intertwined; changes in one parameter generate changes in several other bodily systems. An understanding of the multi-system benefits of pulmonary rehabilitation in the management of COPD is important for patient education and for providing holistic patient-centred care. Moore et al (2012) discovered that the way in which pulmonary rehabilitation was presented to patients influenced the uptake of the programme. For example, the idea of exercise may deter some patients from enrolling on pulmonary rehabilitation programmes; pulmonary 
rehabilitation could then be presented as a choice of activities in which patients can determine which activity they feel comfortable starting with.

Improvement in exercise tolerance was demonstrated in patients with normal exercise capacity (Lan et al 2013), older patients with comorbidities (Gale et al 2011, van Ranst et al 2011) and patients who had experienced exacerbations (Cecins et al 2008). However, significant factors regarding the effectiveness of pulmonary rehabilitation in improving exercise are still to be identified.

It is important for nurses to be aware of the possible enabling factors or barriers to participation in pulmonary rehabilitation, to effectively support patients to undertake pulmonary rehabilitation. Exercise regimens vary according to location and availability of resources, which may complicate the generalisability of research findings. However, this review suggests that the principles underlying pulmonary rehabilitation are the same and emphasises the importance of a person-centred approach when designing pulmonary rehabilitation programmes (Croitoru et al 2013, Gimeno-Santos et al 2014).

COPD has multi-systemic manifestations (Spruit et al 2013). Thus, nurses and patients should be aware of the systemic benefits of pulmonary rehabilitation; the use of multidimensional assessment tools is recommended (Barakat et al 2008). For example, the BODE index (Celli et al 2004), which incorporates the patient's body mass index, airflow obstruction, dyspnoea and exercise capacity, may portray the bodily systems that would benefit from pulmonary rehabilitation activities.

There is conflicting evidence regarding at which stage of COPD patients would benefit from pulmonary rehabilitation (Lan et al 2013) and how soon patients can participate in pulmonary rehabilitation after experiencing exacerbations (Puhan et al 2011). This review considers pulmonary rehabilitation as a collaboration between healthcare professionals and patients, in that patients set their own goals and healthcare professionals support patients to achieve those goals (Halding and Heggdal 2012). For example, a patient who wishes to join a walking group would require different support from one who wants to be able to get around the house and prepare their own meals.

Improvement in breathlessness is a subjective measure that depends on patients' understanding with regard to the diagnosis and prognosis of COPD. This in turn depends on the information patients have access to. According to van Ranst et al (2011), frequent exacerbations and access to healthcare services tend to provide patients with access to education and support; this is associated with improved ability in coping with breathlessness. Pulmonary rehabilitation has been shown to improve breathlessness through patient education, psychological support and breathing retraining.

Improvement in health-related quality of life is also a subjective measure, dependent on the information patients have access to, their age, comorbidities and health beliefs (Riario-Sforza et al 2010). Disease severity and frequency of hospitalisation have been shown to influence health-related quality of life (van Ranst et al 2011). Patient education and psychological wellbeing are mechanisms for the improvement of health-related quality of life in pulmonary rehabilitation.

\section{Limitations}

The goal of pulmonary rehabilitation is a multidisciplinary co-ordinated effort to restore or improve patients' health and achieve the highest possible level of independence (Croitoru et al 2013). The literature review findings cannot be generalised because of the variety of populations and settings in which the studies were conducted. However, the review involved the use of primary studies undertaken worldwide, emphasising the benefits derived from pulmonary rehabilitation and providing evidence that pulmonary rehabilitation can be adapted to benefit different populations in different care settings. This approach aligns well with the COPD Commissioning Toolkit (NHS 2012), which recommends that local pulmonary rehabilitation services should be person-centred. It may be necessary to conduct more qualitative studies regarding the experiences of patients in local communities to establish appropriate pulmonary rehabilitation programmes that meet the needs of individual patients.

\section{Conclusion}

This review identified improvements in exercise tolerance, health-related quality of life and breathlessness as the main benefits of pulmonary rehabilitation in management of patients with COPD. It emphasised the requirement for more qualitative research; and recommended the use of multidimensional assessment tools to identify and address multi-systemic effects of COPD. An understanding of how pulmonary rehabilitation improves the health of patients with COPD enables nurses to engage in effective patient education, encourage the uptake of pulmonary rehabilitation and present pulmonary rehabilitation as an effective multi-systemic intervention NS 


\section{References}

Antoniu SA (2010) Descriptors of dyspnea in obstructive lung diseases. Multidisciplinary Respiratory Medicine. 5, 3, 216-219.

Barakat S, Michele G, George P Nicole V, Guy A (2008) Outpatient pulmonary rehabilitation in patients with chronic obstructive pulmonary disease. International Journal of Chronic Obstructive Pulmonary Disease. 3, 1, 155-162.

Boyatzis RE (1998) Transforming Qualitative Information: Thematic Analysis and Code Development. Sage Publications, London.

Buist S, Anzueto A, Calverley P et al (2006) Global Initiative for Chronic Obstructive Lung Disease. Global Strategy for the Diagnosis, Management, and Prevention of Chronic Obstructive Pulmonary Disease.tinyurl.com/jodyw48 (Lastaccessed: November 17 2015.)

Casaburi R, ZuWallack R (2009) Pulmonary rehabilitation for management of chronic obstructive pulmonary disease. The New England Journal of Medicine. 360, 13, 1329-1335.

Cecins N, Geelhoed E, Jenkins SC (2008) Reduction in hospitalisation following pulmonary rehabilitation in patients with COPD. Australian Health Review. 32, 3, 415-422.

Celli BR, Cote CG, Marin JM et al (2004) The body-mass index airflow obstruction, dyspnea and exercise capacity index in chronic obstructive pulmonary disease. New England Journal of Medicine. 350, 10, 1005-1012.

Corhay J-L, Nguyen Dang D, VanCauwenberge $\mathrm{H}$, Louis $\mathrm{R}$ (2014) Pulmonary rehabilitation and COPD: providing patients a good environment for optimizing therapy. International Journal of Chronic Obstructive Pulmonary Disease. 9, 1, P27-39.

Coventry PA, Bower P, Keyworth C et al (2013) The effect of complex interventions on depression and anxiety in chronic obstructive pulmonary disease: systematic review and meta-analysis. PLoS One. 8, 4, e60532.

Croitoru A, Ioniţă D, Stroescu C et al (2013) Benefits of a 7-week outpatient pulmonary rehabilitation program in COPD patients. Pneumologia. 62, 2, 94-98. de Sousa Pinto JM, Martín-Nogueras AM, Morano MT, Macêdo TE, Arenillas JI, Troosters T (2013) Chronic obstructive pulmonary disease patients' experience with pulmonary rehabilitation: a systematic review of qualitative research. Chronic Respiratory Disease. 10, 3, 141-157.

Gale NS, Duckers JM, Cockcroft JR, Shale DJ, Bolton CE (2011) Does pulmonary rehabilitation address cardiovascular risk factors in patients with COPD? BMC Pulmonary Medicine. doi: 10.1186/1471-2466-11-20.

Gimeno-Santos E, Frei A, Steurer-Stey C et al (2014) Determinants and outcomes of physical activity in patients with COPD: a systematic review. Thorax. 13, 1, 1-9.

Gysels MH, Higginson IJ (2009) Self-management for breathlessness in COPD: the role of pulmonary rehabilitation. Chronic Respiratory Disease. 6, 3, 133-140.

Halding A-G, Heggdal K (2012) Patients' experiences of health transitions in pulmonary rehabilitation. Nursing Inquiry. $19,4,345-356$

Lacasse $Y$, Goldstein R, Lasserson TJ Martin S (2006) Pulmonary rehabilitation for chronic obstructive pulmonary disease. Cochrane Database of Systematic Reviews. Issue 4, CD003793.

Lan CC, Chu WH, Yang MC, Lee $\mathrm{CH}$, Wu YK, Wu CP (2013) Benefits of pulmonary rehabilitation in patients with COPD and normal exercise capacity. Respiratory Care. 58, 9, 1482-1488.

Man WD, Kemp P, Moxham J, Polkey MI (2009) Exercise and muscle dysfunction in COPD: implications for pulmonary rehabilitation. Clinical Science. 117, 8, 281-291.

Marieb E (2011) Essentials of Human Anatomy and Physiology. Tenth edition. Pearson, San Francisco CA.

Martín A, Rodríguez-González Moro J, Izquierdo J, Gobartt E, de Lucas P; VICE Study Group (2008) Health-related quality of life in outpatients with COPD in daily practice: the VICE Spanish study. International Journal of Chronic Obstructive Pulmonary Disease. 3, 4, 683-692.
Moore L, Hogg L, White P (2012) Acceptability and feasibility of pulmonary rehabilitation for COPD: a community qualitative study. Primary Care Respiratory Journal. $21,4,419-424$

National Institute for Health and Care Excellence (2015) Managing Stable COPD. tinyurl.com/nbt4e9v (Last accessed: November 172015.$)$

NHS (2012) COPD Commissioning Toolkit. A Resource for Commissioners. The Stationery Office, London.

Nursing and Midwifery Council (2015) The Code. Professional Standards of Practice and Behaviour for Nurses and Midwives. NMC, London.

O'Donnell DE, Laveneziana P (2006) Physiology and consequences of lung hyperinflation in COPD. European Respiratory Review. 15, 100, 61-67.

Pitta F, Troosters T, Probst VS, Langer D, Decramer M, Gosselink R (2008) Are patients with

COPD more active after pulmonary rehabilitation? Chest $134,2,273-280$.

Plowman SA, Smith DL (2014) Exercise Physiology for Health, Fitness, and Performance. Fourth edition. Lippincott Williams and Wilkins, Baltimore MD.

Puhan M, Gimeno-Santos E, Scharplatz M, Troosters T, Walters EH, Steurer J (2011) Pulmonary rehabilitation following exacerbations of chronic obstructive pulmonary disease. Cochrane Database of Systematic Reviews. Issue 10, CD005305.

Ramli A, Manap RA (2011)

Changes in health related quality of life and exercise tolerance of chronic obstructive pulmonary disease patients following an 8 weeks pulmonary rehabilitation program: the Malaysian experience. International Medical Journal. 18, 4, 322.

Riario-Sforza GG, Yacoub MR Incorvaia C (2010) Pulmonary rehabilitation as evaluated by clinical trials: an overview. Reviews on Recent Clinical Trials. 5, 2, 76-84.

Spruit MA, Singh SJ, Garvey C et al (2013) An official American Thoracic Society/European
Respiratory Society statement: key concepts and advances in pulmonary rehabilitation. American Journal of Respiratory and Critical Care Medicine. 188, 8, el3-e64.

Stav D, Raz M, Shpirer I (2009) Three years of pulmonary rehabilitation: inhibit the decline in airflow obstruction, improves exercise endurance time, and body-mass index, in chronic obstructive pulmonary disease. BMC Pulmonary Medicine. 9,26, 1471-2466.

Troosters T, Gosselink G, Janssens W, Decramer M (2010) Exercise training and pulmonary rehabilitation: new insights and remaining challenges. European Respiratory Review. 19, 115, 24-29.

van Ranst D, Otten H, Meijer JW, van't Hul AJ (2011) Outcome of pulmonary rehabilitation in COPD patients with severely impaired health status. International Journal of Chronic Obstructive Pulmonary Disease. 6, 1, 647-657.

Vincent E, Sewell L (2014) The role of the nurse in pulmonary rehabilitation. Nursing Times. 110, 50, 16-18.

Weldam S, Lammers J, Decates R, Schuurmans M (2013) Daily activities and health-related quality of life in patients with chronic obstructive pulmonary disease: psychological determinants: a cross-sectional study. Health and Quality of Life Outcomes. 11, 190, 2-8.

Williams V, Bruton A, Ellis-Hill C, McPherson K (2010) The effect of pulmonary rehabilitation on perceptions of breathlessness and activity in COPD patients: a qualitative study. Primary Care Respiratory Journal. 19, 1, 45-51.

World Health Organization (2008) COPD Predicted to be Third Leading Cause of Death in 2030. www.who.int/gard/news_events/ World_Health_Statistics_2008/en/ (Lastaccessed: November 17 2015.)

ZuWallack R, Hedges H (2008) Primary care of the patient with chronic obstructive pulmonary disease-part 3: pulmonary rehabilitation and comprehensive care for the patient with chronic obstructive pulmonary disease. American Journal of Medicine. 121, 7 Suppl, S25-32. 